\title{
Abordagem Integrada De Um Incisivo Central Traumatizado - Relato De Caso Clínico
}

An integrated approach of a traumatized central incisor - a case report

Patrícia Inês Chaves Severo', Marcelo Totti², João Ferlini-Filho ${ }^{3}$, Regis Burmeister dos Santos ${ }^{3}$, Simone Bonato Luisi ${ }^{3}$, Maria Carolina Guilherme Erhardt ${ }^{4}$, Fábio Herrmann Coelho-de-Souza ${ }^{4}$

\begin{abstract}
OBJECTIVES: Dental trauma can promote many consequences to dental structure, such as crown, root and surrounding tissues, that depend on the type of trauma. The objective of this case report is to present an integrated approach in a case of dental trauma with endodontic involvement restored with composite resin associated with intracanal fiber post. CASE REPORT: A 12 years-old patient came to dental care service after a dental trauma episode regarding teeth 21 and 22 , being 22 presenting a fail restoration already and 21 presenting extensive fracture and endodontic treatment needs. Tooth 21 showed 2 canals, coronal and root malformation and a mesial perforation. After endodontic treatment and all canals filled, a glass fiber post was luted in the main canal followed by a direct composite restoration. CONCLUSIONS: Therefore, based on this treatment planning, we could rehabilitate the smile of the patient, restoring the shape, function and aesthetics in a conservative way
\end{abstract}

Keywords: Trauma., Aesthetics. Composite resins. Endodontics.

\section{Resumo}

OBJETIVO: O traumatismo alvéolo-dentário pode provocar uma série de consequências às estruturas coronária, radicular e tecidos adjacentes, estando na dependência das características do trauma. O objetivo do presente trabalho é apresentar uma abordagem integrada em um caso clínico de traumatismo com envolvimento endodôntico, sendo realizado o tratamento dos condutos e o selamento da perfuração mesial existente, seguido de reconstrução direta de resina composta associada a retentor intracanal estético. RELATO DE CASO: Paciente de 12 anos compareceu à clínica odontológica com sequelas de trauma recente nos dentes 21 e 22, sendo 022 restaurado previamente de forma deficiente e o 21 com amplo comprometimento coronário e necessidade de tratamento endodôntico. O dente 21 apresentava 2 canais, mal formação coronoradicular e perfuração mesial. Após terapia endodôntica com obturação dos 2 canais e da perfuração, foi cimentado um pino de fibra de vidro no canal principal seguido de reconstrução direta com resina composta. CONSIDERAÇÕES FINAIS: Através do planejamento proposto, obteve-se êxito na reabilitação do paciente, proporcionando a este saúde, forma, função e estética de modo conservador. Em proservação de 6 meses pode se observar sucesso clínico e radiográfico..

Descritores: Trauma. Estética. Resinas compostas. Endodontia.
${ }^{1}$ Especialista em Endodontia - UFRGS

2Mestrando em Dentística/Cariologia - UFRGS

3Professor de Endodontia e Clínica Odontológica - UFRGS

${ }^{4}$ Professor de Dentística e Clínica Odontológica - UFRGS

Correspondência: Marcelo Totti

Endereço: Rua Ramiro Barcelos 2492, Porto Alegre, RS, Brasil, 90035-003

E-mail: tottipstpoa@hotmail.com.br

\section{Introdução}

O traumatismo alvéolo-dentário pode afetar as estruturas bucais levando a diversas conseqüências que podem variar desde uma pequena fratura de esmalte até a perda do elemento dentário (CARDOSO e CARVALHO, 2002). Quando o trauma ocorre na dentição decídua, pode produzir sequelas nos germes dentários da dentição permanente, acarretando, frequentemente, mal formações dentárias, sejam elas coronárias, coronorradiculares ou radiculares (MELLO-MOURA et al., 2009). Assim sendo, dentes desenvolvidos de germes com essa mal formação podem sofrer alterações no número e morfologia dos condutos radiculares, podendo representar uma dificuldade no eventual manejo endodôntico de tais elementos (ALTUN et al., 2008; MELLO-MOURA et al., 2009; SIQUEIRA et al., 2013; TSURUMACHI et al., 2012).

Nos casos de traumatismo alvéolo-dentários em que a polpa é atingida de modo irreversível, o tratamento endodôntico é a solução que permite a manutenção do dente em boca com condições de ser restaurado adequadamente. Para isso, diversas técnicas são propostas na literatura. Todavia, canais com formação atípica, perfurações, obstruções e curvaturas são considerados desafios para a Endodontia (SIQUEIRA et al. 2013; SUSHIL et al., 2013; NAHSAN et al., 2012; ALTUN et al., 2008).

Fraturas coronárias provenientes de trauma requerem uma abordagem clínica que está na dependência do comprometimento tecidual causado pelo evento traumático, podendo variar desde a colagem de um fragmento dentário autógeno, até a reconstrução coronária ou ainda a reabilitação por implantes e próteses fixas (SUSHIL et al., 2013; TSURUMACHI et al., 2012). Quando estes eventos ocorrem em áreas de grande comprometimento estético, 0 tratamento reabilitador é ainda mais desafiador para o cirurgiãodentista. Nesse contexto, o mercado odontológico apresenta uma série de materiais estéticos para que se possam reproduzir as características dos dentes naturais, buscando a mimetização da restauração com a estrutura dentária. Com a evolução das resinas compostas e da Odontologia restauradora adesiva, criou-se a possibilidade de resolver casos com extensas fraturas através de restauração direta com resina composta e sistemas adesivos, podendo estar associada ainda a retentores intrarradiculares estéticos, como os pinos de fibra de vidro, por exemplo. Esta técnica, além de ter baixo custo e não necessitar de muitas sessões clínicas, é capaz de oferecer um excelente resultado estético, satisfazendo os requisitos de forma, função e estética, agradando tanto ao cirurgiãodentista quanto ao paciente (SUSHIL et al., 2013; NAHSAN et al., 2012). 
O presente trabalho tem por objetivo apresentar um caso clinico de reconstrução coronária com resina composta direta associada a pino intracanal em um dente permanente jovem que sofreu trauma. O presente caso clínico busca ilustrar e auxiliar o clínico na tomada de decisão e melhor planejamento baseado em evidência para casos de trauma de difícil manejo endodôntico e restaurador.

\section{Caso Clínico}

Paciente do gênero masculino, com 12 anos de idade, chegou à clínica odontológica com sequelas de trauma nos elementos dentários 21 e 22, sendo que o dente 22 apresentava restauração prévia deficiente, enquanto o dente 21 mostrava amplo comprometimento coronário (fratura e selamento provisório) e necessidade de tratamento endodôntico (figuras 1 e 2).

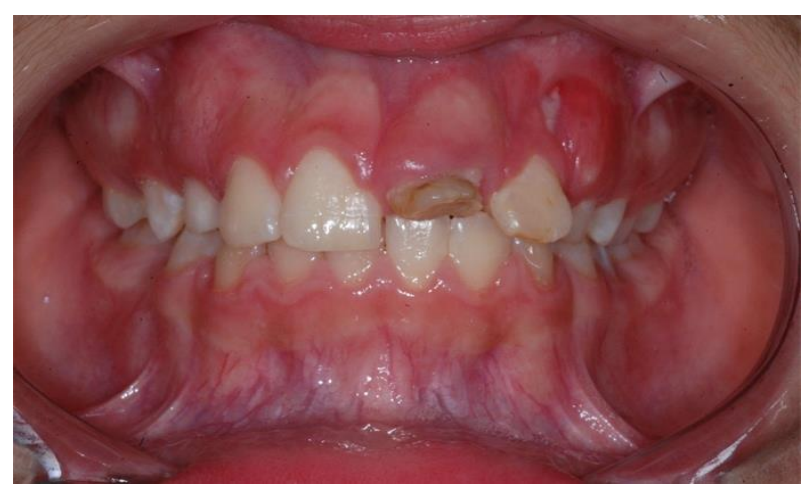

Figura 1 - Aspecto inicial, vista frontal, destacando a destruição coronária do dente 21 e restauração deficiente no dente 22.

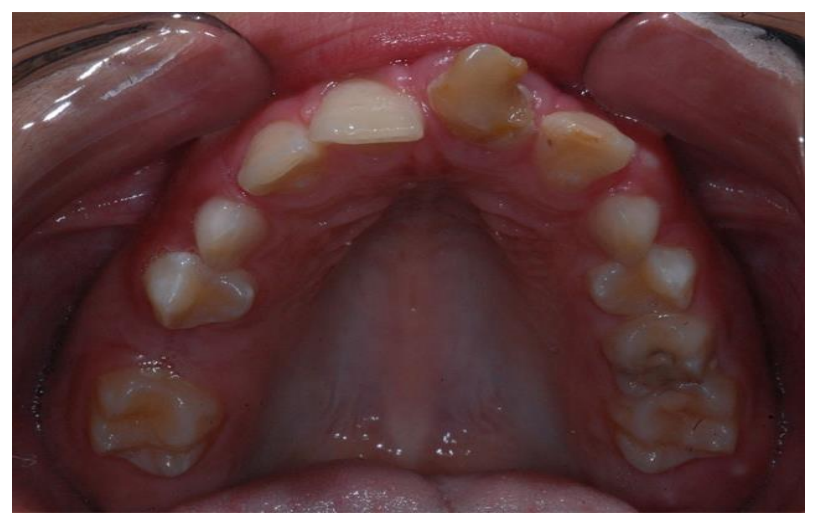

Figura 2 - Aspecto inicial, vista oclusal, destacando o posicionamento do dente 21 .

A partir do exame radiográfico, verificou-se que o dente 21 apresentava 2 condutos radiculares (1 principal e 1 colateral), perfuração mesial e mal formação coronorradicular, além de área radiolúcida mesial compatível com lesão inflamatória lateral (figura 3). O paciente relatou histórico de traumatismo na dentição decídua - o que justifica as alterações anatômicas coronorradiculares - e novo trauma recente na dentição permanente seguido de necrose pulpar e fratura coronária extensa. $\mathrm{O}$ plano de tratamento elaborado visou a manutenção do dente 21. Para isso, decidiu-se realizar o tratamento endodôntico e a reabilitação estética com restauração direta de resina composta associada a retentor intrarradicular estético (pino de fibra de vidro), visando retenção e resistência

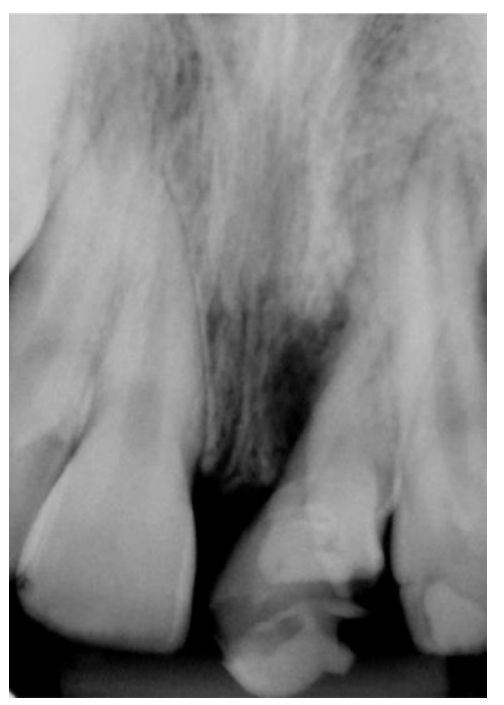

Figura 3 - Aspecto radiográfico inicial. Observar anatomia atípica e lesão inflamatória mesial.

coronorradicular (NOTHDURFT et al., 2008; SALAMEH et al., 2008; D'ARCANGELO et al., 2008). A terapia endodôntica foi realizada pela técnica coroa-ápice (figura 4) e obturação dos condutos radiculares pela técnica da condensação lateral. Observou-se a obturação dos dois canais radiculares paralelos (principal e colateral) e da perfuração com guta percha e cimento endodôntico (Endofill Dentsply, York, PA, USA). Ocorreu sobre-obturação lateral mesial, devido à dificuldade de controlar a inserção dos cones de guta percha acessórios (figura 5). Todavia, optou-se pela proservação do caso, observando-se, em um primeiro momento, a resposta clínica do paciente (ausência de sintomatologia) e, posteriormente, o resultado radiográfico (desejável formação de tecido ósseo reparador) (conforme necessidade futura, seria realizado acesso cirúrgico para remoção dos excessos de material obturador endodôntico).

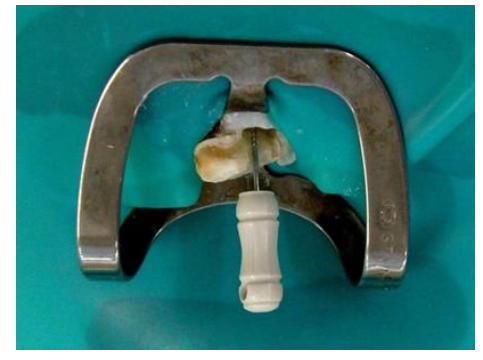

Figura 4 - Localização dos canais para tratamento endodôntico.

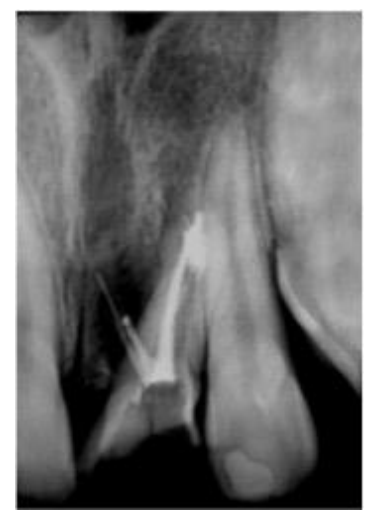

Figura 5 - Tratamento endodôntico concluído com desobturação parcial do canal principal e sobre-obturação lateral mesial. 
Após a terapia endodôntica foi realizado o tratamento restaurador, com a reconstrução estética do dente 21 associada ao pino de fibra de vidro. Para tanto, obteve-se uma impressão em alginato para a confecção de modelo de estudo em gesso (figura 6) para enceramento diagnóstico do caso (figura 7) e confecção de guia de silicona para conduzir a restauração (técnica estratificada), bem como escolha das cores de resina composta a serem utilizadas na restauração.
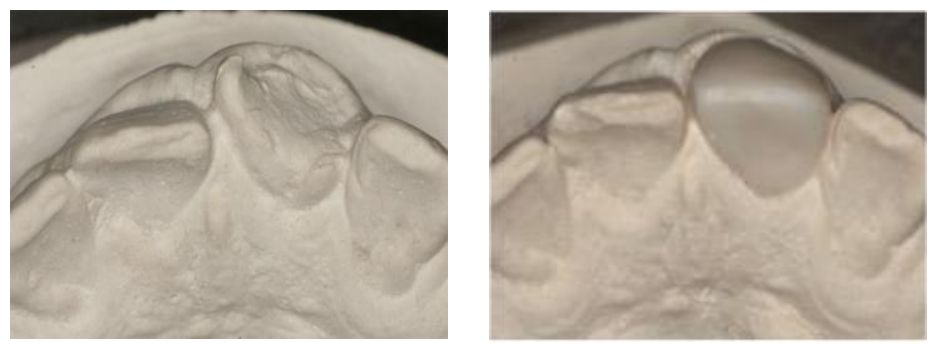

Figuras 6 e 7 - Modelo de estudo (pós-endodontia) e encerado (reanatomização)

Foi então realizada uma desobturação parcial do canal radicular principal, através de brocas Gates-Gliden e Largo, mantendo $4 \mathrm{~mm}$ de obturação endodôntica para selamento apical (figura 5). Após, um pino de fibra de vidro (White Post DC1 - FGM, Joinvile, SC, Brasil) (figura 8) foi testado no canal principal. Após a realização do isolamento do campo operatório, o pino foi cimentado com cimento resinoso convencional de presa dual (AllCem, FGM), seguido de reconstrução direta com resina composta seguindo a técnica incremental com guia de silicona, para auxílio da conformação do incremento relativo ao esmalte palatino. O sistema adesivo empregado no caso foi o Scothbond Multiuso (3M ESPE, Sumaré, SP, Brasil), e a resina composta utilizada foi a Filtek Z350 XT (3M ESPE), nas cores A3D, B3D, A3E, A2E e CT. O dente 22 teve sua restauração substituída, utilizando os mesmos materiais supracitados. O acabamento das restaurações foi realizado com pontas diamantadas 3195F e 3118F, lâminas de bisturi número 12 seguido de tiras de lixa para as faces proximais, discos sof-lex série laranja (3M ESPE) para a face vestibular, pontas siliconizadas abrasivas (Enhance - Dentsply, York, PA, USA) e roda de feltro com pasta diamantada Enamelize (Cosmedent, Chicago, IL, USA) para 0 polimento. Nas figuras 9 e 10 pode-se visualizar o aspecto final da reabilitação estética (dentes 21 e 22), em vista frontal e lateral, em que se pode observar a devolução da forma anatômica, cor e um melhor alinhamento dentário para o dente 21. Decorridos 6 meses da conclusão do caso (proservação), observou-se sucesso clínico e radiográfico (figura 11). As restaurações dos dentes 21 e 22 mantiveram suas características iniciais e o tratamento endodôntico apresentou mutismo clínico e sinais de reparo ósseo na lesão lateral mesial, como se pode perceber na figura 11.

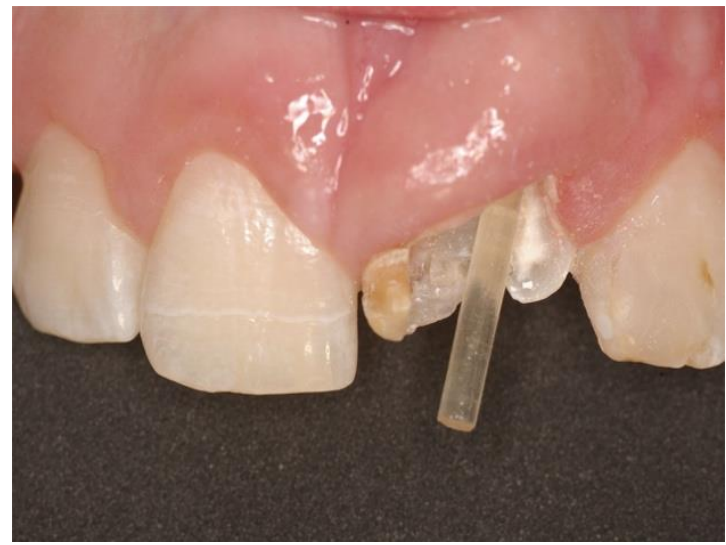

Figura 8 - Prova do pino de fibra de vidro prévio à cimentação e inclinação acentuada do pino

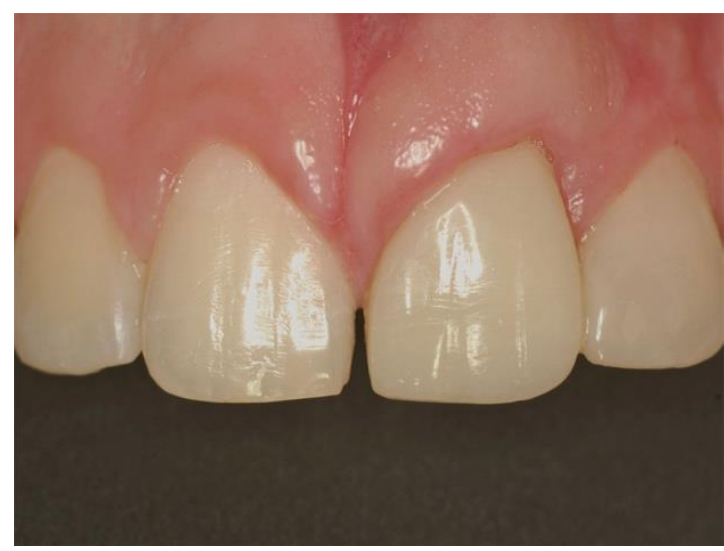

Figura 9 - Caso concluído, vista frontal final (reconstrução do 21 e substituição da restauração do 22 em resina composta) .

\section{Discussão}

Traumatismos que afetam dentes anteriores são comuns em crianças e podem atingir as dentições decídua, mista ou permanente. Quando esse tipo de injúria ocorre sobre a dentição permanente, o tratamento e a manutenção dos dentes envolvidos podem representar um desafio para o clínico (ALTUN et al., 2008).

O caso clínico descrito no presente trabalho diz respeito a um paciente jovem com histórico de trauma na dentição decídua, o que pode justificar a mal formação apresentada pelo dente 21(MELLOMOURA et al., 2009). Após novo episódio de trauma, agora na dentição permanente, houve extensa fratura coronária envolvendo esmalte e dentina com comprometimento pulpar. Apesar da dificuldade anatômica, o tratamento endodôntico foi planejado para permitir a manutenção do dente em boca com condições de ser restaurado adequadamente. No dente 21 havia um canal principal, um canal colateral paralelo ao primeiro, e uma perfuração mesial. A obturação dos canais apresentou-se com boa qualidade (canais principal e colateral), mas com sobre-obturação da perfuração mesial. Optou-se pela proservação do caso, guardando uma eventual cirurgia para-

Rev. Fac. Odontol. Porto Alegre, v. 59, n.1 , p. 6-10, jan./jun., 2018. 
endodôntica para um segundo momento, se necessário. Após um período de 6 meses, a proservação mostrou um promissor início de reparo ósseo na lesão mesial, indicando possível sucesso do tratamento, apesar da presença da sobre-obturação.

Nas últimas décadas, o avanço da Odontologia adesiva permitiu que uma grande variedade de materiais estéticos entrasse no mercado odontológico, oferecendo ao clínico diversas opções para alcançar o tratamento de excelência no restabelecimento de saúde, função e estética. Nos casos de traumatismos dentários com extensas fraturas, a reconstrução destes elementos por meio de restauração direta de resina composta associada a retentores estéticos intrarradiculares encontra respaldo na literatura (ALTUN et al., 2008; GARBIN et al., 2010; BITTER e KIELBASSA, 2007; DA SILVA et al., 2010). Essa técnica descrita apresenta como vantagens um baixo custo (poucas sessões clínicas e não envolve procedimentos indiretos), preservação do dente em boca, da estrutura dental remanescente e devolução funcional e estética adequadas (CONCEIÇÂO e CONCEIÇÂO, 2012).

A utilização de retentores intrarradiculares é capaz de conferir maior retenção, resistência (NOTHDURFT et al., 2008; D'ARCANGELO et al., 2008 SALAMEH et al., 2008) e possibilitar uma restauração com boa adaptação marginal, aspectos que são considerados importantes para preservar a qualidade da obturação dos condutos radiculares( BITTER e KIELBASSA, 2007.). Outras características importantes dos pinos de fibra de vidro são: a adesividade aos cimentos resinosos, e seu módulo de elasticidade semelhante ao da dentina, o que confere maior distribuição das forças oclusais no longo eixo do dente e possibilita que, quando exista falha, essa seja mais favorável à manutenção do dente e ao reparo da restauração( ALTUN et al., 2008; BITTER e KIELBASSA, 2007; DA SILVA et al., 2010.).

O presente relato de caso clínico de tratamento endodôntico atípico e restauração extensa de resina composta associada a pino intracanal demonstra uma possibilidade de planejamento e tratamento para esse tipo de situação clínica, buscando uma solução adequada, conservadora e que permitisse a manutenção do dente traumatizado em boca. Num período de tempo de 6 meses, as restaurações realizadas estavam satisfatórias e, radiograficamente, pode se observar a cicatrização da lesão inflamatória mesial. Todavia, estudos de avaliação clínica longitudinal desse tipo de procedimento devem ser conduzidos para elucidar o verdadeiro comportamento clínico ao longo do tempo.

\section{Conclusão}

O planejamento proposto para o caso clínico contemplou todos os aspectos necessários para a reabilitação do sorriso do paciente. Apesar das características anatômicas, a terapia endodôntica possibilitou a manutenção do dente em boca, e a restauração direta realizada atingiu os requisitos morfológicos e funcionais. Assim sendo, considera-se que o tratamento executado para o caso em questão foi capaz de proporcionar ao paciente saúde e estética de maneira conservadora.

\section{Referências}

ALTUN, C.: TOZUM, T.F.; GUVEN, G. Multidisciplinary approach to the rehabilitation of a crown fracture with glass-fibre-reinforced composite: a case report. J Can. Dent. Assoc, Ottawa v. 74, no. 4, p.363-366, May. 2008.

BITTER, K.; KIELBASSA, A.M. Post-endodontic restorations with adhesively luted fiber-reinforced composite post systems: a review. Am J dent, Memphis V. 20, no.6, p 353-360, Dec. 2007.

CARDOSO, M.; DE CARVALHO ROCHA, M.J. Traumatized primary teeth in children assisted at the Federal University of Santa Catarina, Brazil. Dent traumatol, Copenhagen V.18, no. 3, p. 129-133, May. 2002.

CONCEIÇÃO, A.B; CONCEIÇÃO, E.N. Emprego dos pinos de fibra em Odontologia. In: COELHO-DE-SOUZA, F.H, (Ed.). Tratamentos clínicos integrados em Odontologia. Rio de Janeiro, Brasil: Revinter, 2012. Cap 16 - Emprego dos pinos de fibra em odontologia, p 287-304.

DA SILVA, N.R; RAPOSO, L.H; VERSLUIS, A; FERNANDES-NETO, A.J; SOARES, C.J. The effect of post, core, crown type, and ferrule presence on the biomechanical behavior of endodontically treated bovine anterior teeth. J prosthet dent, St. Louis V. 104, no. 5, p. 306317, Nov. 2010.

D'ARCANGELO, C.; DE ANGELIS, F.; VADINI, M.; ZAZZERONI, S.; CIAMPOLI, C.; D'AMARIO, M. In vitro fracture resistance and deflection of pulpless teeth restored with fiber posts and prepared for veneers. J endod, Baltimore v. 34, no. 7, p. 838-841, Jul 2008.

GARBIN, C.A.; SPAZZIN, A.O.; MEIRA-JUNIOR, A.D.; LORETTO, S.C.; LYRA, A.M.; BRAZ, R. Biomechanical behaviour of a fractured maxillary incisor restored with direct composite resin only or with different post systems. Int endod j, Oxford v.43, no. 12, p. 1098-1107, Dec 2010

MELLO-MOURA, A.C.; BONINI, G.A.; SUGA, S.S.; NAVARRO, R.S.; WANDERLEY, M.T. Multidisciplinary approach on rehabilitation of primary teeth traumatism repercussion on the permanent successor: 6-year follow-up case report. J Indian Soc Pedod Prev Dent v. 27, no.2, p. 125-130, Apr-Jun 2009.

NAHSAN, F.P.; MONDELLI, R.F.; FRANCO, E.B.; NAUFEL, F.S. UEDA, J.K.; SCHMITT, V.L.; BASSEGIO, W. Clinical strategies for esthetic excellence in anterior tooth restorations: understanding color and composite resin selection. $\mathbf{J}$ appl oral sci, v. 20 , no. 2, p. 151156, Mar. 2012.

NOTHDURFT, F.P.; SEIDEL, E.; GEBHART, F.; NAUMANN, M.; MOTTER, P.J.; POSPIECH, P.R. The fracture behavior of premolar teeth with class II cavities restored by both direct composite restorations and endodontic post systems. J dent, Guildford v. 36, no. 6, p. 444-449, Jun 2008.

Rev. Fac. Odontol. Porto Alegre, v. 59, n.1, p. 6-10, jan./jun., 2018. 
SALAMEH, Z.; SORRENTINO, R.; OUNSI, H.F.; SADIG, W.; ATIYEH, F.; FERRARI, M. The effect of different full-coverage crown systems on fracture resistance and failure pattern of endodontically treated maxillary incisors restored with and without glass fiber posts. $\mathbf{J}$ endod. Baltimore v.34, no.7, p.842-846, Jul 2008.

SIQUEIRA, M.B.; GOMES, M.C.; OLIVEIRA, A.C.; MARTINS, C.C.; GRANVILLE-GARCIA, A.F.; PAIVA, S.M. Predisposing Factors for Traumatic Dental Injury in Primary Teeth and Seeking of Post-trauma Care. Braz dent j, Ribeirão preto v. 24, no. 6, p. 647-654, May 2013.

SUSHIL, K.C.; RAO, A.; SHEILA, K.; HANUMANTH, R.G.

Multidisciplinary Approach in Management of Fractured Central Incisor through Composite Plug Stabilization - A Case Report. J int oral health, Ahmedabad v. 5, no. 1, p. 79-82, Jan-Feb 2013.

TSURUMACHI, T,; MATSUMOTO, S.; KOBAYASHI, Y.; OHARA, K.; SUZUKI, Y.; OGISO, B. Esthetic and endodontic management of a deep crown-root fracture of a maxillary central incisor. $\mathbf{J}$ oral sci, Tokyo v.54, no. 4, p. 359- 362, Dec. 2012.

Rev. Fac. Odontol. Porto Alegre, v. 59, n.1 , p. 6-10, jan./jun., 2018. 